\title{
How do supermarket 'healthy' range ready meals compare with the 'standard' equivalent?
}

\author{
J. Bradbury and N. Day \\ School of Applied Sciences, University of Huddersfield, Huddersfield HD1 3DH
}

The UK market for 'ready meals and main meal accompaniments' was worth over $£ 1.5$ billion in the year up to January $2016^{(1)}$. Ready meals have been characterised as 'unhealthy', being high in fat, saturated fat and salt ${ }^{(3-4)}$. Most ready meals are supermarket own-brand $^{(2)}$, and there are usually several ranges within each supermarket's ready meal offering, including a 'healthy' version of the standard product. A recent study that compared the nutrient profile of meals from the 'luxury', 'standard', 'value' and 'healthy' ranges found that the 'healthy' meals were more likely to be lower in energy, fat, saturated fat, and salt ${ }^{(4)}$. However, only six meal types were examined. The aim of this study was to compare the price, energy and nutrient content of 'healthy' range ready meals with their 'standard' equivalent.

Data available online from five UK supermarkets (Sainsbury's, Tesco, Waitrose, Asda, Morrisons) were collected during March 2016 for own-brand 'healthy' and 'standard' range chilled, one person, ready meals. The 'healthy' range(s) were identified first and then ready meals within the range were matched with a comparable meal from the supermarket's 'standard' range. If there was no comparable match the 'healthy' product was not included. Information on energy, total and saturated fats, total carbohydrates and total sugars, fibre, protein and salt content per $100 \mathrm{~g}$ was collected, as were pack weight and price. The energy and nutrient content per pack was calculated; contributions to Reference Intakes (RI) were calculated as a percentage of the RIs. As variables were not normally distributed, medians (interquartile range, IQR) are reported and non-parametric statistical tests were conducted.

Included in the analysis were 180 ready meals. Pack weights ranged from $175 \mathrm{~g}$ to $500 \mathrm{~g}$, with the 'healthy' range meals significantly lighter than the 'standard' meals $[400 \mathrm{~g}(20 \mathrm{~g}) v s .430 \mathrm{~g}(50 \mathrm{~g}), p<0.001)$. Prices ranged from $£ 2.00$ to $£ 3.90$; there was a significant difference in price per pack ['healthy' $£ 2.50(£ 0.68)$ vs. 'standard' $£ 2.50(£ 0.70), p=0.023$; this difference was explained by the higher priced 'healthy' meals for Morrisons [£2.32 for all 'healthy' meals vs. $£ 2.17(£ 0 \cdot 25)$ for 'standard', $p=0.039]$ and Waitrose [£3.30 $(£ 0.80)$ vs. $£ 2.50(£ 0.75), p=0.009)$. When price was expressed per $100 \mathrm{~g}$, the 'healthy' meals were more expensive $[£ 0 \cdot 66(£ 0 \cdot 20)$ vs. $£ 0.59(£ 0.17), p<0.001]$. The 'healthy' range meals were lower than the 'standard' meals in energy, total and saturated fats, protein and salt, expressed as per $100 \mathrm{~g}$ and as per pack. Based on a ready meal contributing one third of daily energy and nutrient intake, when compared against one third of the relevant RIs, energy and nutrients were significantly different for both ranges (all $p<0 \cdot 001$ ).

\begin{tabular}{|c|c|c|c|c|c|c|c|c|c|c|c|c|c|c|}
\hline \multirow[b]{3}{*}{ Energy $(\mathrm{kJ})$} & \multicolumn{14}{|c|}{ Median (IQR) } \\
\hline & \multicolumn{2}{|c|}{$\begin{array}{l}\text { Healthy } \\
\mathrm{g} / 100 \mathrm{~g}\end{array}$} & \multicolumn{2}{|c|}{$\begin{array}{l}\text { Standard } \\
\mathrm{g} / 100 \mathrm{~g}\end{array}$} & \multirow{2}{*}{$\begin{array}{l}P \\
<0.001\end{array}$} & \multicolumn{2}{|c|}{$\begin{array}{l}\begin{array}{l}\text { Healthy } \\
\text { g/pack }\end{array} \\
\end{array}$} & \multicolumn{2}{|c|}{$\begin{array}{l}\text { Standard } \\
\text { g/pack }\end{array}$} & \multirow{2}{*}{$\begin{array}{l}P \\
<0 \cdot 001\end{array}$} & \multicolumn{2}{|c|}{$\begin{array}{l}\text { Healthy } \\
\% \text { RI }\end{array}$} & \multicolumn{2}{|c|}{$\begin{array}{l}\text { Standard } \\
\% \mathrm{RI}\end{array}$} \\
\hline & 402 & (80) & 547 & (145) & & 372 & (66) & 545 & (174) & & 18 & (3) & 27 & (9) \\
\hline Total fat & $2 \cdot 0$ & $(0.9)$ & 4.9 & $(2 \cdot 3)$ & $<0.001$ & 7.8 & (3.2) & $20 \cdot 8$ & $(10 \cdot 2)$ & $<0.001$ & 11 & (5) & 30 & (15) \\
\hline Saturated fat & 0.8 & $(0.5)$ & 1.8 & $(1.9)$ & $<0.001$ & 3.0 & (1.9) & $7 \cdot 6$ & $(7.9)$ & $<0.001$ & 15 & (10) & 38 & (39) \\
\hline Carbohydrate & $12 \cdot 1$ & $(4.5)$ & 13.8 & $(6.8)$ & 0.062 & $47 \cdot 2$ & $(16 \cdot 2)$ & 56.5 & $(26 \cdot 7)$ & 0.001 & 18 & (6) & 22 & (10) \\
\hline Sugars & $2 \cdot 4$ & $(1.7)$ & $2 \cdot 1$ & $(1.8)$ & 0.671 & $9 \cdot 0$ & $(6 \cdot 5)$ & 8.7 & $(7.5)$ & 0.674 & 10 & (7) & 10 & (8) \\
\hline Dietary fibre ${ }^{\dagger}$ & 1.7 & $(0.8)$ & 1.8 & $(1 \cdot 1)$ & 0.903 & 6.8 & $(2.7)$ & 7.2 & $(5 \cdot 5)$ & 0.179 & 23 & (9) & 24 & (18) \\
\hline Protein & $6 \cdot 5$ & $(1.7)$ & $7 \cdot 3$ & $(2 \cdot 0)$ & $<0.001$ & $25 \cdot 2$ & $(7 \cdot 1)$ & $30 \cdot 5$ & $(10 \cdot 0)$ & $<0.001$ & 50 & (14) & 61 & (20) \\
\hline Salt & 0.38 & $(0.08)$ & 0.50 & $(0.15)$ & $<0.001$ & 1.47 & $(0.37)$ & $2 \cdot 25$ & $(0.76)$ & $<0.001$ & 25 & (6) & 38 & (13) \\
\hline
\end{tabular}

†compared with the UK dietary reference value of $30 \mathrm{~g} / \mathrm{d}$.

In this study of a wide range of meal types, the 'healthy' range meals had a healthier nutrient profile than the equivalent 'standard' meals, although they were also more expensive (per $100 \mathrm{~g}$ ). This may deter low income consumers from purchasing the healthier option $^{(5)}$.

1. Chilled Food Association. http://www.chilledfood.org/wp-content/uploads/2015/07/Kantar-Chilled-Prepared-Foods-Jan-2016-uploaded-18-1-16. pdf (accessed April 2016).

2. Euromonitor International (2015) http://www.euromonitor.com/ready-meals-in-the-united-kingdom/report (accessed April 2016).

3. Howard S, Adams J \& White M (2012) BMJ 345, e7607.

4. Remnant J \& Adams J (2015) Appetite 92, 36-42.

5. Darmon N \& Drewnowski A (2015) Nutr Rev 73, 643-660. 